\title{
Offloading data traffic via cognitive small cells with wireless powered user equipments
}

\author{
Ding $X u^{*}$ (D) and Qun Li
}

\begin{abstract}
This paper investigates data traffic offloading by considering a third-party cognitive small cell with wireless powered user equipments (UEs) providing data traffic offloading service to a primary macrocell. The cognitive small cell is assumed to use remaining resources for its own purpose provided that the quality of service (QoS) of the primary macrocell is satisfied. It is assumed that the small cell UEs (SUEs) are wirelessly powered and can harvest energy from the RF signals transmitted by the macrocell UEs (MUEs) as well as the RF signals transmitted by the small cell BS (SBS). Under the assumption that the successive interference cancellation (SIC) decoder is available or not available at the SBS, iterative optimization-based data traffic offloading schemes are proposed to maximize the SUE sum rate provided that the required minimum MUE sum rate is satisfied. It is shown that the proposed data traffic offloading schemes are effective in improving the performance of the MUEs and providing transmission opportunities for the wireless powered SUEs.
\end{abstract}

Keywords: Data traffic offloading, Energy harvesting, Cognitive radio

\section{Introduction}

Data traffic offloading is an effective way to address the network overloading issue for overloaded cellular networks due to unprecedented increase in data traffic [1]. The quality of service (QoS) of the users in overloaded networks cannot be satisfied, while data traffic offloading can offload part of the data traffic load off the overloaded networks, and thus, the QoS of the users can be improved.

Meanwhile, the recently proposed cognitive radio (CR) technology is able to address the spectrum scarcity problem by allowing the secondary users who have no licensed spectrum band to share the spectrum bands licensed to the primary users [2]. In order to protect the licensed primary users, the activities of the secondary users cannot affect the QoS of the primary users. Various problems in $\mathrm{CR}$ networks have been studied, such as resource allocation [3-8] and security issues $[9,10]$. Generally, if the primary users cannot benefit from allowing the secondary users to transmit using their licensed spectrum bands, the primary users have no incentive to do so. Therefore, in the situation where the primary networks are overloaded

*Correspondence: xuding@ieee.org

Nanjing University of Posts and Telecommunications, Nanjing, China and the secondary networks are light loaded, some primary users can direct their data traffic to the secondary networks for better service experience, and as a reward for the secondary networks, they can access the licensed spectrum bands as long as the QoS of the primary users is satisfied. Particularly, CR networks can form small cells to deal with the data traffic offloaded from the primary networks.

Energy harvesting is a promising technology that can provide perpetual energy to wireless equipments [11]. Especially, for small wireless equipments such as wireless sensors or small portable equipments, energy harvesting through radio frequency (RF) signals is very attractive. Naturally, CR and energy harvesting can be jointly considered and designed. Specifically, with energyharvesting capability, CR equipments can be powered by green energy sources and RF signals. Thus, energy harvesting provides a sustainable power supply to the energy-constrained CR networks. On the other hand, with CR capability, energy-harvesting wireless networks can explore new available spectrum for overcoming the issue of limited available spectrum.

Therefore, for wireless powered secondary user equipments (UEs) in CR networks, they can harvest energy from RF signals transmitted from the primary UEs as well 
as from a dedicated power station. For the CR networks that offer data traffic offloading service to the primary networks, the wireless powered secondary UEs can harvest energy while the primary UEs are transmitting and transmit using the harvested energy while the QoS of the primary UEs is satisfied and the secondary UEs are allowed to transmit. Such data traffic offloading for the primary UEs via $C R$ networks with wireless powered secondary UEs can achieve a win-win situation for the primary UEs and the secondary UEs. However, how to optimize time allocation between energy harvesting and data transmission, how to determine which primary UEs are offloaded to the CR network, and how to control the transmit powers of the secondary UEs and the primary UEs remain unknown. This motivates the work in this paper.

This paper considers a third-party cognitive small cell with wireless powered secondary UEs that provides data traffic offloading service to a primary macrocell. As long as the QoS of the primary macrocell is satisfied, the cognitive small cell can use the remaining time and frequency resources for its own purpose. Specifically, successive interference cancellation (SIC) decoder is assumed to be available at the macrocell BS (MBS), while the small cell BS (SBS) is assumed to be equipped or not equipped with SIC decoder. It is assumed that the small cell UEs (SUEs) are wirelessly powered and can harvest energy from the RF signals transmitted by the macrocell UEs (MUEs) as well as the RF signals transmitted by the SBS. With or without SIC decoder at the SBS, we aim to optimize data traffic offloading, time, and power allocation for maximizing the SUE sum rate under the constraint that the required minimum MUE sum rate is achieved. Such optimization problems are highly nonlinear nonconvex, and thus, optimal solutions are unknown. To solve the optimization problems, we propose iterative optimization-based schemes to iteratively optimize data traffic offloading, time, and power allocation.

The main contributions and results of this paper are summarized as follows.

- We consider a secondary small cell with wireless powered SUEs to provide data traffic offloading service to MUEs in a primary macrocell and formulate the problem of optimizing data traffic offloading, time, and power allocation for maximizing the SUE sum rate under the required minimum MUE sum rate.

- By assuming that SIC decoder is available or not available at the SBS, we propose an iterative optimization-based scheme to solve the optimization problem by iteratively optimizing data traffic offloading, time, and power allocation.

- We show that the proposed data traffic offloading schemes are effective in improving the performance of the MUEs and providing transmission opportunities for the SUEs. Specifically, the MUE sum rate with offloading is shown to be significantly higher than that without offloading. It is also shown that equipping SIC decoder at the SBS can increase both the SUE sum rate and the MUE sum rate compared to the case without SIC decoder at the SBS. In addition, it is shown that increasing the transmit power limit of the MUEs is beneficial to both the MUEs and the SUEs.

The remainder of the paper is organized as follows. Section 2 surveys works related to this paper. Section 3presents the system model. Section 4 presents the data traffic offloading scheme without SIC decoder at the SBS. Section 5 presents the data traffic offloading scheme with SIC decoder at the SBS. Section 6 verifies the proposed data traffic offloading schemes using extensive simulation results. Section 7 concludes the paper.

\section{Related work}

So far, data traffic offloading in wireless networks has been researched a lot. In [12], a two-level offloading scheme that takes the network load and interference conditions into account in small cell networks was proposed. In [13], a learning mechanism-based fair auction scheme for data offloading in small cell networks was proposed. In [14], an optimal energy-efficient offloading scheme based on the auction theory was proposed. In [15], a network-assisted user-centric WiFi offloading scheme in a heterogeneous network was proposed. In [16], the efficiency of the opportunistic and the delayed WiFi offloading schemes was analyzed. In [17], the problem of joint BS switching, resource allocation, and data traffic offloading was investigated. Note that all the works in [12-17] on data traffic offloading did not consider CR and energy-harvesting capabilities for the target data traffic offloading networks.

The works that considered data traffic offloading through CR networks include [18-21]. In [18], the authors proposed to deploy cognitive small cells to offload the data traffic from the long-term evolution (LTE) network. In the work, the cognitive small cells were assumed to belong to the LTE network's operator. In [19], a flexible CR functional architecture was proposed for offloading data traffic from the LTE network using the TV whitespaces and was mapped to the LTE network architecture. In [20], the authors considered traffic offloading through heterogeneous networks, where the offloaded users are treated as the secondary users and the users in the heterogeneous networks are treated as the primary users. In the work, Stackelberg game was used to optimize the utilities of the secondary users and the primary users. In [21], the cognitive small cells were assumed to offload users from the congested macrocells and the authors explored stochastic 
geometry to investigate the load of each cells and the effects of different offloading techniques. Note that, different from this paper, all the works in [18-21] assumed that the secondary users are powered by constant energy sources.

Our paper is also related to the work on energyharvesting-based wireless networks. In [22], the authors investigated simultaneous wireless information and power transfer for non-regenerative multiple input multiple-output orthogonal frequency-division multiplexing (MIMO-OFDM) relaying systems and proposed two protocols to maximize the throughput. In [23], an energy-efficient resource allocation scheme was proposed for an OFDM based full-duplex distributed antenna system with energy-harvesting capability. In [24], an iterative subchannel and power allocation scheme was proposed for a heterogeneous cloud small cell network with simultaneous wireless information and power transfer. In [25], a wireless powered communication network with group energy cooperation was considered and the resource allocation was optimized to maximize the weighted sum rate and minimize the power consumption. In [26], the problem of joint user association and power allocation in a millimeter wave ultra dense network with energy-harvesting base stations was investigated and an iterative gradient-based algorithm was proposed. In [27], the capacity region of a multiple access channel with energy-harvesting transmitters and energy cooperation was derived. Note that, as the works in [22-27] are not for CR, providing data traffic offloading service to the primary users is not of concern.

The works on energy-harvesting-based CR networks include [28-32]. In [28], the time and energy allocation problem for $\mathrm{CR}$ multiple access networks with energy harvesting was formulated as a Stackelberg game, and then, the Stackelberg equilibrium was derived. In [29], a distributed channel selection strategy was proposed for a multichannel CR system with energy-harvesting secondary users. In [30], the optimal power control and time allocation for CR networks with wireless powered secondary users to maximize the sum rate of the secondary users under the interference power constraint at the primary user was derived. In [31], the wireless powered secondary users were assumed to relay the signals from the primary users and energy-efficient scheduling and power control algorithms were proposed. In [32], the secondary users were assumed to cooperate with the wireless powered primary users in exchange for transmission opportunities and the resource allocation schemes to maximize the sum rate of the secondary users under the minimum rate constraint at the primary users were proposed. It is noted that, although the works in [28-32] considered to guarantee or improve the performance of the primary users, unlike this paper, they did not consider that the SUs can provide offloading service to the primary users. To our best knowledge, there is no work on the topic of data traffic offloading considering both $\mathrm{CR}$ and energy-harvesting capabilities yet.

\section{System model}

An uplink macrocell network with $M$ MUEs and a MBS licensed with a narrow spectrum band is considered ${ }^{1}$, as shown in Fig. 1. Meanwhile, an uplink small cell network with $K$ wireless powered SUEs and a SBS with no licensed spectrum band is considered to be in the coverage area of the macrocell network. It is assumed that the MUEs can be served by the MBS or offloaded to be served by the SBS. As long as the performance of the macrocell network is guaranteed, the small cell network can use the spectrum licensed to the macrocell network for its own purpose.

We assume that all the channels are block-fading, i.e., the channel power gains are constant in each transmission block and change independently. The channel power gains from the MUE $m$ to the MBS, between the SUE $k$ and the SBS, from the MUE $m$ to the SBS, and from the MUE $m$ to the SUE $k$ are denoted by $h_{m}^{p}, h_{k}^{s}, h_{m}^{p s}$, and $h_{m, k}$, respectively. We assume that perfect channel state information (CSI) on these channel power gains is available at a central control unit (CCU) which is responsible for making the data traffic offloading decision. Usually, the MBS can be the CCU. The CSI of the links from the MUEs to the MBS and the links between the SUEs to the SBS can be obtained by classic channel estimation methods, while the CSI of the links from the MUEs to the SBS and from the MUEs to the SUEs can be obtained by cooperation between the macrocell and the small cell as proposed in [33].

The transmission time for each transmission block denoted by $T$ is assumed to be divided into three slots.

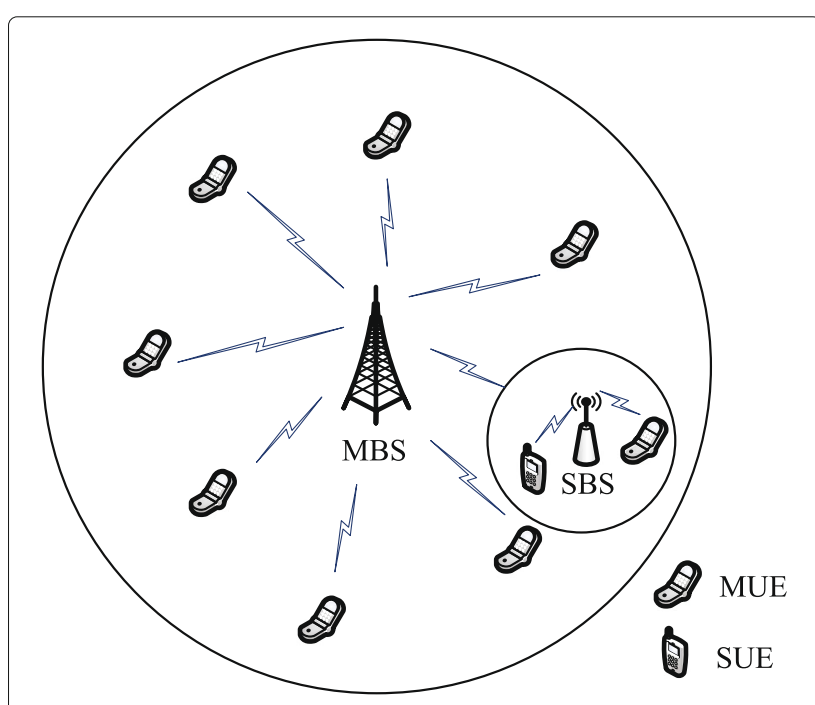

Fig. 1 System model 
The first slot is for MUE data communication with time $\tau_{0}$. It is assumed that the SUEs can harvest energy from the received signals transmitted by the MUEs in this slot. The second slot with time $\tau_{1}$ is for the SBS to broadcast energy wirelessly to the SUEs with transmit power $p_{\mathrm{SBS}}^{s}$. The third slot with time $\tau_{2}$ is for the SUEs to use harvested energy from the former two slots to transmit data to the SBS.

Let $p_{m}^{p}$ and $p_{k}^{s}$ denote the transmit power of the MUE $m$ in the first slot and the transmit power of the SUE $k$ in the third slot, respectively. We denote $\boldsymbol{p}^{p}=\left[p_{1}^{p}, \ldots, p_{M}^{p}\right]^{T}$ and $\boldsymbol{p}^{s}=\left[p_{1}^{s}, \ldots, p_{K}^{s}\right]^{T}$. The transmit power of the MUEs is restricted as $p_{m}^{p} \leq P_{\max }^{p}$ for $m=1, \ldots, M$ due to hardware limits. The energy harvested by the SUE $k$ during the first and the second slots is given by

$$
E_{k}=\zeta \tau_{0} \sum_{m=1}^{M} p_{m}^{p} h_{m, k}+\zeta \tau_{1} p_{\mathrm{SBS}}^{s} h_{k}^{s},
$$

where $\zeta$ denotes the energy-harvesting efficiency. Thus, the consumed energy during the third slot for the SUE $k$ cannot exceed the energy harvested during the first and the second slots as given by

$$
p_{k}^{s} \tau_{2} \leq \zeta \tau_{0} \sum_{m=1}^{M} p_{m}^{p} h_{m, k}+\zeta \tau_{1} p_{\mathrm{SBS}}^{s} h_{k}^{s} .
$$

Let $\alpha_{m} \in\{0,1\}$ and $\beta_{m} \in\{0,1\}$ denote whether the MUE $m$ is connected to the MBS and the SBS, respectively, where $\alpha_{m}=1$ denotes that the MUE $m$ is connected to the MBS and vice versa, while $\beta_{m}=1$ denotes that the MUE $m$ is offloaded to the SBS and vice versa. It is assumed that each MUE can be connected to either the MBS or the SBS, i.e., $\alpha_{m}+\beta_{m} \leq 1$, for $m=1, \ldots, M$. We denote $\boldsymbol{\alpha}=\left[\alpha_{1}, \ldots, \alpha_{M}\right]^{T}$ and $\boldsymbol{\beta}=\left[\beta_{1}, \ldots, \beta_{M}\right]^{T}$.

In order to guarantee the QoS of the macrocell network, the minimum MUE sum rate is required to be larger than a threshold as given $b^{2}$

$$
R_{p}\left(\tau_{0}, \boldsymbol{\alpha}, \boldsymbol{p}^{p}\right)+R_{\mathrm{ps}}\left(\tau_{0}, \boldsymbol{\beta}, \boldsymbol{p}^{p}\right) \geq R_{\min },
$$

where $R_{p}\left(\tau_{0}, \boldsymbol{\alpha}, \boldsymbol{p}^{p}\right)$ is the sum rate of the MUEs connected to the MBS, $R_{\mathrm{ps}}\left(\tau_{0}, \boldsymbol{\beta}, \boldsymbol{p}^{p}\right)$ is the sum rate of the MUEs offloaded to the SBS, and $R_{\min }$ is the required minimum MUE sum rate.

Our aim is to maximize the SUE sum rate denoted by $R_{s}\left(\tau_{2}, \boldsymbol{p}^{S}\right)$. We assume that the MBS is powerful and equipped with SIC decoder while the SBS is less powerful and may or may not be equipped with SIC decoder. Thus, $R_{p}\left(\tau_{0}, \boldsymbol{\alpha}, \boldsymbol{p}^{p}\right)$ can be expressed as

$$
R_{p}\left(\tau_{0}, \boldsymbol{\alpha}, \boldsymbol{p}^{p}\right)=\frac{\tau_{0}}{T} \ln \left(1+\frac{\sum_{m=1}^{M} \alpha_{m} p_{m}^{p} h_{m}^{p}}{\sigma^{2}}\right),
$$

and the exact expressions of $R_{\mathrm{ps}}\left(\tau_{0}, \boldsymbol{\beta}, \boldsymbol{p}^{p}\right)$ and $R_{s}\left(\tau_{2}, \boldsymbol{p}^{s}\right)$ depend on whether SIC decoder is available at the SBS.
On the one hand, if SIC decoder is not available at the SBS, the expressions of $R_{\mathrm{ps}}\left(\tau_{0}, \boldsymbol{\beta}, \boldsymbol{p}^{p}\right)$ and $R_{s}\left(\tau_{2}, \boldsymbol{p}^{s}\right)$ can be written as

$$
\begin{aligned}
R_{\mathrm{ps}}^{\mathrm{NSIC}}\left(\tau_{0}, \boldsymbol{\beta}, \boldsymbol{p}^{p}\right)= & \frac{\tau_{0}}{T} \sum_{m=1}^{M} \ln \\
& \times\left(1+\frac{\beta_{m} p_{m}^{p} h_{m}^{\mathrm{ps}}}{\sigma^{2}+\sum_{m^{\prime}=1, m^{\prime} \neq m}^{M} \beta_{m^{\prime}} p_{m^{\prime}}^{p}{ }^{\prime^{\mathrm{pS}}}}\right),
\end{aligned}
$$

and

$$
R_{s}^{\mathrm{NSIC}}\left(\tau_{2}, \boldsymbol{p}^{s}\right)=\frac{\tau_{2}}{T} \sum_{k=1}^{K} \ln \left(1+\frac{p_{k}^{s} h_{k}^{s}}{\sigma^{2}+\sum_{k^{\prime}=1, k^{\prime} \neq k}^{K} p_{k^{\prime}}^{s} h_{k^{\prime}}^{s}}\right),
$$

respectively. On the other hand, if SIC decoder is available at the SBS, the expressions of $R_{\mathrm{ps}}\left(\tau_{0}, \boldsymbol{\beta}, \boldsymbol{p}^{p}\right)$ and $R_{s}\left(\tau_{2}, \boldsymbol{p}^{s}\right)$ can be written as

$$
R_{\mathrm{ps}}^{\mathrm{SIC}}\left(\tau_{0}, \boldsymbol{\beta}, \boldsymbol{p}^{p}\right)=\frac{\tau_{0}}{T} \ln \left(1+\frac{\sum_{m=1}^{M} \beta_{m} p_{m}^{p} h_{m}^{\mathrm{ps}}}{\sigma^{2}}\right),
$$

and

$$
R_{s}^{\mathrm{SIC}}\left(\tau_{2}, \boldsymbol{p}^{s}\right)=\frac{\tau_{2}}{T} \ln \left(1+\frac{\sum_{k=1}^{K} p_{k}^{s} h_{k}^{s}}{\sigma^{2}}\right),
$$

respectively.

\section{Offloading scheme without SIC decoder at the SBS}

This section investigates the data traffic offloading scheme when SIC decoder is not available at the SBS. The optimization problem is formulated as

$$
\begin{aligned}
& \max _{\tau_{0}, \tau_{1}, \tau_{2}, \boldsymbol{\alpha}, \boldsymbol{\beta}, \boldsymbol{p}^{p}, \boldsymbol{p}^{s}} R_{s}^{\mathrm{NSIC}}\left(\tau_{2}, \boldsymbol{p}^{s}\right) \\
& \text { s.t. } \tau_{0}+\tau_{1}+\tau_{2} \leq T, \tau_{0} \geq 0, \tau_{1} \geq 0, \tau_{2} \geq 0, \\
& \alpha_{m} \in\{0,1\}, \beta_{m} \in\{0,1\}, \forall m, \\
& \alpha_{m}+\beta_{m} \leq 1, \forall m, \\
& 0 \leq p_{m}^{p} \leq P_{\max }^{p}, \forall m, \\
& 0 \leq p_{k}^{s} \tau_{2} \leq \zeta \tau_{0} \sum_{m=1}^{M} p_{m}^{p} h_{m, k}+\zeta \tau_{1} p_{\mathrm{SBS}}^{s} h_{k}^{s}, \forall k, \\
& R_{p}\left(\tau_{0}, \boldsymbol{\alpha}, \boldsymbol{p}^{p}\right)+R_{\mathrm{ps}}^{\mathrm{NSIC}}\left(\tau_{0}, \boldsymbol{\beta}, \boldsymbol{p}^{p}\right) \geq R_{\min } .
\end{aligned}
$$

The above problem is a highly nonconvex nonlinear problem, and thus, the optimal solution is hard to obtain. We solve the above problem by iteratively optimizing $\tau_{0}, \tau_{1}, \tau_{2}$ with given $\boldsymbol{\alpha}, \boldsymbol{\beta}, \boldsymbol{p}^{p}, \boldsymbol{p}^{s}$, optimizing $\boldsymbol{\alpha}, \boldsymbol{\beta}$ with given $\tau_{0}, \tau_{1}, \tau_{2}, \boldsymbol{p}^{p}, \boldsymbol{p}^{s}$, and optimizing $\boldsymbol{p}^{p}, \boldsymbol{p}^{s}$ with given $\tau_{0}, \tau_{1}, \tau_{2}, \boldsymbol{\alpha}, \boldsymbol{\beta}$. 
With given $\boldsymbol{\alpha}, \boldsymbol{\beta}, \boldsymbol{p}^{p}, \boldsymbol{p}^{s}$, the problem in (9) reduces to the following problem to optimize $\tau_{0}, \tau_{1}$, and $\tau_{2}$ as given by

$$
\begin{aligned}
& \max _{\tau_{0}, \tau_{1}, \tau_{2}} R_{s}^{\mathrm{NSIC}}\left(\tau_{2}, \boldsymbol{p}^{s}\right) \\
& \text { s.t. } \tau_{0}+\tau_{1}+\tau_{2} \leq T, \tau_{0} \geq 0, \tau_{1} \geq 0, \tau_{2} \geq 0 \\
& p_{k}^{s} \tau_{2}-\zeta \tau_{0} \sum_{m=1}^{M} p_{m}^{p} h_{m, k}-\zeta \tau_{1} p_{\mathrm{SBS}}^{s} h_{k}^{s} \leq 0, \forall k \\
& \tau_{0} \geq \frac{R_{\min } T}{C_{p}}
\end{aligned}
$$

where $C_{p}=\ln \left(1+\frac{\sum_{m=1}^{M} \alpha_{m} p_{m}^{p} h_{m}^{p}}{\sigma^{2}}\right)+\sum_{m=1}^{M} \ln$ $\left(1+\frac{\beta_{m} p_{m}^{p} h_{m}^{\mathrm{ps}}}{\sigma^{2}+\sum_{m^{\prime}=1, m^{\prime} \neq m}^{\beta_{m^{\prime}} p_{m^{\prime}}^{\prime^{\prime}} \mathrm{ps}_{m^{\prime}}^{\mathrm{ps}}}}\right)$. It is observed that the above problem belongs to the linear programming and thus can be solved efficiently by linear programming methods such as the simplex method [34].

With given $\tau_{0}, \tau_{1}, \tau_{2}, \boldsymbol{p}^{p}, \boldsymbol{p}^{s}$, the problem to optimize $\boldsymbol{\alpha}, \boldsymbol{\beta}$ is given by

$$
\begin{aligned}
\max _{\boldsymbol{\alpha}, \boldsymbol{\beta}} & R_{s}^{\mathrm{NSIC}}\left(\tau_{2}, \boldsymbol{p}^{s}\right) \\
\text { s.t. } & \alpha_{m} \in\{0,1\}, \beta_{m} \in\{0,1\}, \forall m, \\
& \alpha_{m}+\beta_{m} \leq 1, \forall m, \\
& R_{p}\left(\tau_{0}, \boldsymbol{\alpha}, \boldsymbol{p}^{p}\right)+R_{\mathrm{ps}}^{\mathrm{NSIC}}\left(\tau_{0}, \boldsymbol{\beta}, \boldsymbol{p}^{p}\right) \geq R_{\min } .
\end{aligned}
$$

The objective function of the above problem does not depend on $\boldsymbol{\alpha}, \boldsymbol{\beta}$. Thus, the above problem can be solved by checking whether the problem is feasible by solving the following problem as given by

$$
\begin{aligned}
\max _{\boldsymbol{\alpha}, \boldsymbol{\beta}} & R_{p}\left(\tau_{0}, \boldsymbol{\alpha}, \boldsymbol{p}^{p}\right)+R_{\mathrm{ps}}^{\mathrm{NSIC}}\left(\tau_{0}, \boldsymbol{\beta}, \boldsymbol{p}^{p}\right) \\
\text { s.t. } & \alpha_{m} \in\{0,1\}, \beta_{m} \in\{0,1\}, \forall m, \\
& \alpha_{m}+\beta_{m} \leq 1, \forall m .
\end{aligned}
$$

It is noted that the problem in (20) is infeasible if the obtained maximum objective function value in (24) is smaller than $R_{\min }$. The problem in (24) belongs to integer programming and thus is hard to be solved. Here, we propose a heuristic scheme as follows. Initially, we set $\alpha_{m}=0, \beta_{m}=0$ for all $m=1, \ldots, M$. Then, the MUEs are sequentially decided to be connected to the MBS or the SBS by selecting the one that provides higher objective function value in (24). Since the sequence of the MUEs has great impact on the performance of the heuristic scheme, we randomly generate the sequence of the MUEs several times and then pick the one that provides the highest objective function value in (24). The algorithm to solve the problem in (24) is summarized in Algorithm 1.

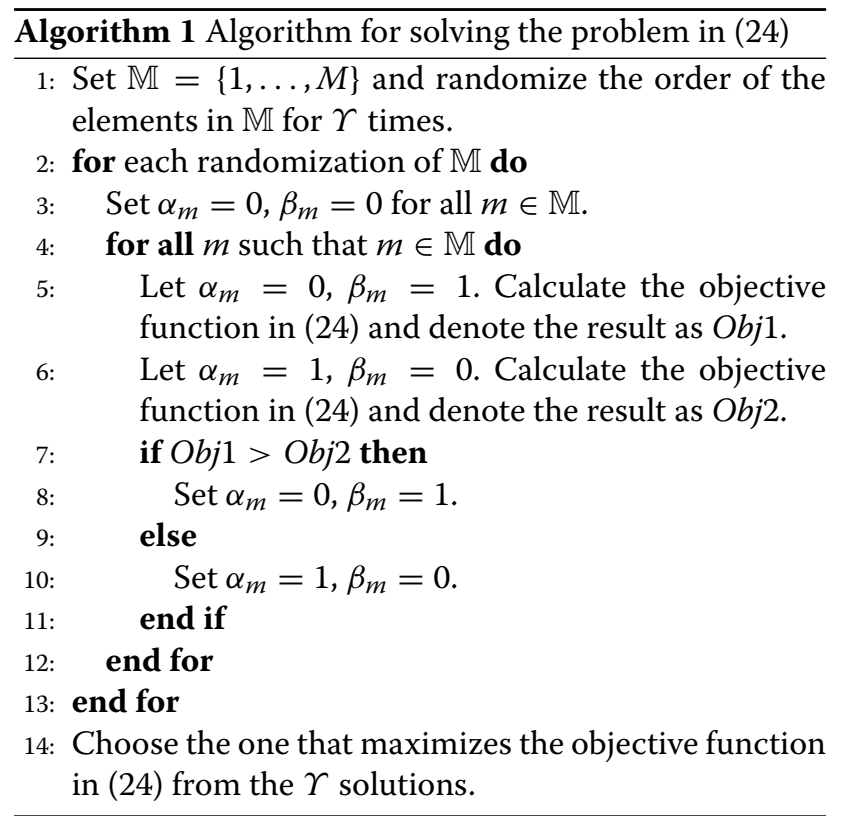

With given $\tau_{0}, \tau_{1}, \tau_{2}, \boldsymbol{\alpha}, \boldsymbol{\beta}$, the problem to optimize $\boldsymbol{p}^{p}, \boldsymbol{p}^{s}$ is given by

$$
\begin{aligned}
\max _{\boldsymbol{p}^{p}, \boldsymbol{p}^{s}} R_{s}^{\mathrm{NSIC}}\left(\tau_{2}, \boldsymbol{p}^{s}\right) \\
\text { s.t. } 0 \leq p_{m}^{p} \leq P_{\mathrm{max}}^{p}, \forall m, \\
0 \leq p_{k}^{s} \leq \frac{\zeta \tau_{0} \sum_{m=1}^{M} p_{m}^{p} h_{m, k}+\zeta \tau_{1} p_{\mathrm{SBS}}^{s} h_{k}^{s}}{\tau_{2}}, \forall k \\
\quad R_{p}\left(\tau_{0}, \boldsymbol{\alpha}, \boldsymbol{p}^{p}\right)+R_{\mathrm{ps}}^{\mathrm{NSIC}}\left(\tau_{0}, \boldsymbol{\beta}, \boldsymbol{p}^{p}\right) \geq R_{\min } .
\end{aligned}
$$

We solve the above problem by iteratively optimizing $\boldsymbol{p}^{s}$ with the given $\boldsymbol{p}^{p}$ and optimizing $\boldsymbol{p}^{p}$ with the given $\boldsymbol{p}^{s}$. With the given $\boldsymbol{p}^{p}$, we optimize $\boldsymbol{p}^{s}$ as

$$
\begin{aligned}
& \max _{p^{s}} R_{s}^{\mathrm{NSIC}}\left(\tau_{2}, \boldsymbol{p}^{s}\right) \\
& \text { s.t. } 0 \leq p_{k}^{s} \leq \frac{\zeta \tau_{0} \sum_{m=1}^{M} p_{m}^{p} h_{m, k}+\zeta \tau_{1} p_{\mathrm{SBS}}^{s} h_{k}^{s}}{\tau_{2}}, \forall k .
\end{aligned}
$$

The above problem can be solved by iteratively optimizing one variable with other variables being fixed. With the given $p_{1}^{s}, \ldots, p_{k-1}^{s}, p_{k+1}^{s}, \ldots, p_{K}^{s}$, the objective function in (31) is a function of $p_{k}^{s}$ and is denoted by $f_{k}^{s}\left(p_{k}^{s}\right)$. The first derivative of $f_{k}^{s}\left(p_{k}^{s}\right)$ can be obtained as

$$
\begin{aligned}
\frac{d f_{k}^{s}\left(p_{k}^{s}\right)}{d p_{k}^{s}}= & \frac{\tau_{2} h_{k}^{s}}{T\left(\sigma^{2}+\sum_{l=1}^{K} p_{l}^{s} h_{l}^{s}\right)} \\
& \times\left(1-\sum_{k^{\prime}=1, k^{\prime} \neq k}^{K} \frac{p_{k^{\prime}}^{s} h_{k^{\prime}}^{s}}{\sigma^{2}+\sum_{l=1, l \neq k^{\prime}}^{K} p_{l}^{s} h_{l}^{s}}\right) .
\end{aligned}
$$


It can be verified that $\frac{\tau_{2} h_{k}^{s}}{T\left(\sigma^{2}+\sum_{l=1}^{K} p_{l}^{s} h_{l}^{s}\right)}$ is positive, and the remaining part of $\frac{d f_{k}^{s}\left(p_{k}^{s}\right)}{d p_{k}^{s}}$ is a strictly increasing function of $p_{k}^{s}$. Thus, the solution to $\frac{d f_{k}^{s}\left(p_{k}^{s}\right)}{d p_{k}^{s}}=0$ denoted by $p_{k}^{s}=x_{k}$ is unique. The function $f_{k}^{s}\left(p_{k}^{s}\right)$ is an increasing function of $p_{k}^{s}$ if $x_{k} \leq 0$, is a decreasing function of $p_{k}^{s}$ if $x_{k} \geq P_{\max }^{s}$, and decreases as $p_{k}^{s}$ increases and turns to increase when $p_{k}^{s}$ is beyond $x_{k}$ if $0<x_{k}<P_{\max }^{s}$. Based on the above analysis, the solution of $p_{k}^{s}$ is $p_{k}^{s}=P_{\max }^{s}$ if $x_{k} \leq 0$, is $p_{k}^{s}=0$ if $x_{k} \geq P_{\max }^{s}$, is $p_{k}^{s}=0$ if $0<x_{k}<P_{\max }^{s}, f_{k}^{s}(0)>f_{k}^{s}\left(P_{\max }^{s}\right)$, and is $p_{k}^{s}=P_{\max }^{s}$ if $0<x_{k}<P_{\max }^{s}, f_{k}^{s}(0) \leq f_{k}^{s}\left(P_{\max }^{s}\right)$. In summary, the solution of $p_{k}^{s}$ is given by

$$
p_{k}^{s}= \begin{cases}P_{\max }^{s}, & x_{k} \leq 0, \\ P_{\max }^{s}, & 0<x_{k}<P_{\max }^{s}, f_{k}^{s}(0) \leq f_{k}^{s}\left(P_{\max }^{s}\right), \\ 0, & \text { otherwise. }\end{cases}
$$

With given $\boldsymbol{p}^{s}$, we optimize $\boldsymbol{p}^{p}$ as

$$
\begin{aligned}
\max _{\boldsymbol{p}^{p}} & R_{s}^{\mathrm{NSIC}}\left(\tau_{2}, \boldsymbol{p}^{s}\right) \\
\text { s.t. } 0 \leq p_{m}^{p} \leq P_{\max }^{p}, \forall m, & \\
& \sum_{m=1}^{M} p_{m}^{p} h_{m, k} \geq \frac{p_{k}^{s} \tau_{2}-\zeta \tau_{1} p_{\mathrm{SBS}}^{s} h_{k}^{s}}{\zeta \tau_{0}}, \forall k, \\
& R_{p}\left(\tau_{0}, \boldsymbol{\alpha}, \boldsymbol{p}^{p}\right)+R_{\mathrm{ps}}^{\mathrm{NSIC}}\left(\tau_{0}, \boldsymbol{\beta}, \boldsymbol{p}^{p}\right) \geq R_{\min } .
\end{aligned}
$$

The objective function of the above problem does not depend on $\boldsymbol{p}^{p}$. Thus, the above problem can be solved by checking whether the problem is feasible by solving the following problem as given by

$$
\begin{aligned}
\max _{\boldsymbol{p}^{p}} & R_{p}\left(\tau_{0}, \boldsymbol{\alpha}, \boldsymbol{p}^{p}\right)+R_{\mathrm{ps}}^{\mathrm{NSIC}}\left(\tau_{0}, \boldsymbol{\beta}, \boldsymbol{p}^{p}\right) \\
\text { s.t. } & \leq p_{m}^{p} \leq P_{\max }^{p}, \forall m, \\
& \sum_{m=1}^{M} p_{m}^{p} h_{m, k} \geq \frac{p_{k}^{s} \tau_{2}-\zeta \tau_{1} p_{\mathrm{SBS}}^{s} h_{k}^{s}}{\zeta \tau_{0}}, \forall k .
\end{aligned}
$$

It is noted that the problem in (35) is infeasible if the obtained maximum objective function value in (39) is smaller than $R_{\mathrm{min}}$. The problem in (39) is solved in a heuristic way. First, without considering the constraint in (41), the problem in (39) is solved in what follows. Since $R_{p}\left(\tau_{0}, \boldsymbol{\alpha}, \boldsymbol{p}^{p}\right)$ is an increasing functions of $\boldsymbol{p}^{p}$. Thus, the optimal $p_{m}^{p}$ is given as $p_{m}^{p}=P_{\max }^{p}$ for $m \in\left\{m \mid \alpha_{m}=1\right.$, $m=1, \ldots, M\}$. For the optimal $\left\{p_{m}^{p}, m \in\left\{m \mid \beta_{m}=1\right.\right.$, $m=1, \ldots, M\}\}$ of the problem in (39), we iteratively optimize one variable with other variables being fixed similar to the problem in (31) and obtain the solution of $p_{m}^{p}$ as given by

$$
p_{m}^{p}= \begin{cases}P_{\max }^{p}, & y_{m} \leq 0 \\ P_{\max }^{p}, & 0<y_{m}<P_{\max }^{p}, f_{m}^{p}(0) \leq f_{m}^{p}\left(P_{\max }^{p}\right), \\ 0, & \text { otherwise }\end{cases}
$$

where $f_{m}^{p}\left(p_{m}^{p}\right)$ denotes the function $R_{\mathrm{ps}}^{\mathrm{NSIC}}\left(\tau_{0}, \boldsymbol{\beta}, \boldsymbol{p}^{p}\right)$ of $p_{m}^{p}$ with other variables being fixed, and $y_{m}$ is the solution to the following equation as given by

$$
1=\sum_{m^{\prime} \in \mathbb{M}_{\beta}, m^{\prime} \neq m} \frac{p_{m^{\prime}}^{p} h_{m^{\prime}}^{\mathrm{ps}}}{\sigma^{2}+\sum_{l \in \mathbb{M}_{\beta}, l \neq m^{\prime}} p_{l}^{p} h_{l}^{\mathrm{ps}}},
$$

where $\mathbb{M}_{\beta}=\left\{m \mid \beta_{m}=1, m=1, \ldots, M\right\}$. The details are omitted here for brevity. Then, we check whether the constraint in (41) is satisfied. If the constraint in (41) is violated, then we select the MUE from the set $\left\{m \mid \beta_{m}=1\right.$, $\left.p_{m}^{p}=0, m=1, \ldots, M\right\}$ that decreases the objective function in (39) to the smallest extent if its transmit power is set to $P_{\max }^{p}$. The above procedure continues until the constraint in (41) is satisfied. The algorithm to solve the problem in (27) is summarized in Algorithm 2.

The overall data traffic offloading scheme without SIC decoder at the SBS is listed in Algorithm 3.

\section{Offloading scheme with SIC decoder at the SBS}

This section investigates the data traffic offloading scheme when SIC decoder is available at the SBS. The optimization problem is formulated as

$$
\begin{aligned}
& \max _{\tau_{0}, \tau_{1}, \tau_{2}, \boldsymbol{\alpha}, \boldsymbol{\beta}, \boldsymbol{p}^{p}, \boldsymbol{p}^{s}} R_{s}^{\mathrm{SIC}}\left(\tau_{2}, \boldsymbol{p}^{s}\right) \\
& \text { s.t. } \tau_{0}+\tau_{1}+\tau_{2} \leq T, \tau_{0} \geq 0, \tau_{1} \geq 0, \tau_{2} \geq 0, \\
& \alpha_{m} \in\{0,1\}, \beta_{m} \in\{0,1\}, \forall m, \\
& \alpha_{m}+\beta_{m} \leq 1, \forall m, \\
& 0 \leq p_{m}^{p} \leq P_{\max }^{p}, \forall m, \\
& 0 \leq p_{k}^{s} \tau_{2} \leq \zeta \tau_{0} \sum_{m=1}^{M} p_{m}^{p} h_{m, k}+\zeta \tau_{1} p_{\mathrm{SBS}}^{s} h_{k}^{s}, \forall k, \\
& R_{p}\left(\tau_{0}, \boldsymbol{\alpha}, \boldsymbol{p}^{p}\right)+R_{\mathrm{ps}}^{\mathrm{SIC}}\left(\tau_{0}, \boldsymbol{\beta}, \boldsymbol{p}^{p}\right) \geq R_{\min } .
\end{aligned}
$$

Similar to the problem in (9), the above problem is solved by iteratively optimizing $\tau_{0}, \tau_{1}, \tau_{2}$ with given $\boldsymbol{\alpha}, \boldsymbol{\beta}, \boldsymbol{p}^{p}, \boldsymbol{p}^{s}$, optimizing $\boldsymbol{\alpha}, \boldsymbol{\beta}$ with given $\tau_{0}, \tau_{1}, \tau_{2}, \boldsymbol{p}^{p}, \boldsymbol{p}^{s}$, and optimizing $\boldsymbol{p}^{p}, \boldsymbol{p}^{s}$ with given $\tau_{0}, \tau_{1}, \tau_{2}, \boldsymbol{\alpha}, \boldsymbol{\beta}$.

With given $\boldsymbol{\alpha}, \boldsymbol{\beta}, \boldsymbol{p}^{p}, \boldsymbol{p}^{s}$, we optimize $\tau_{0}, \tau_{1}$, and $\tau_{2}$ as given by

$$
\begin{aligned}
& \max _{\tau_{0}, \tau_{1}, \tau_{2}} R_{s}^{\mathrm{SIC}}\left(\tau_{2}, \boldsymbol{p}^{s}\right) \\
& \text { s.t. } \tau_{0}+\tau_{1}+\tau_{2} \leq T, \tau_{0} \geq 0, \tau_{1} \geq 0, \tau_{2} \geq 0 \\
& p_{k}^{s} \tau_{2}-\zeta \tau_{0} \sum_{m=1}^{M} p_{m}^{p} h_{m, k}-\zeta \tau_{1} p_{\mathrm{SBS}}^{s} h_{k}^{s} \leq 0, \forall k \\
& \tau_{0} \geq \frac{R_{\min } T}{C_{p}^{\mathrm{SIC}}}
\end{aligned}
$$




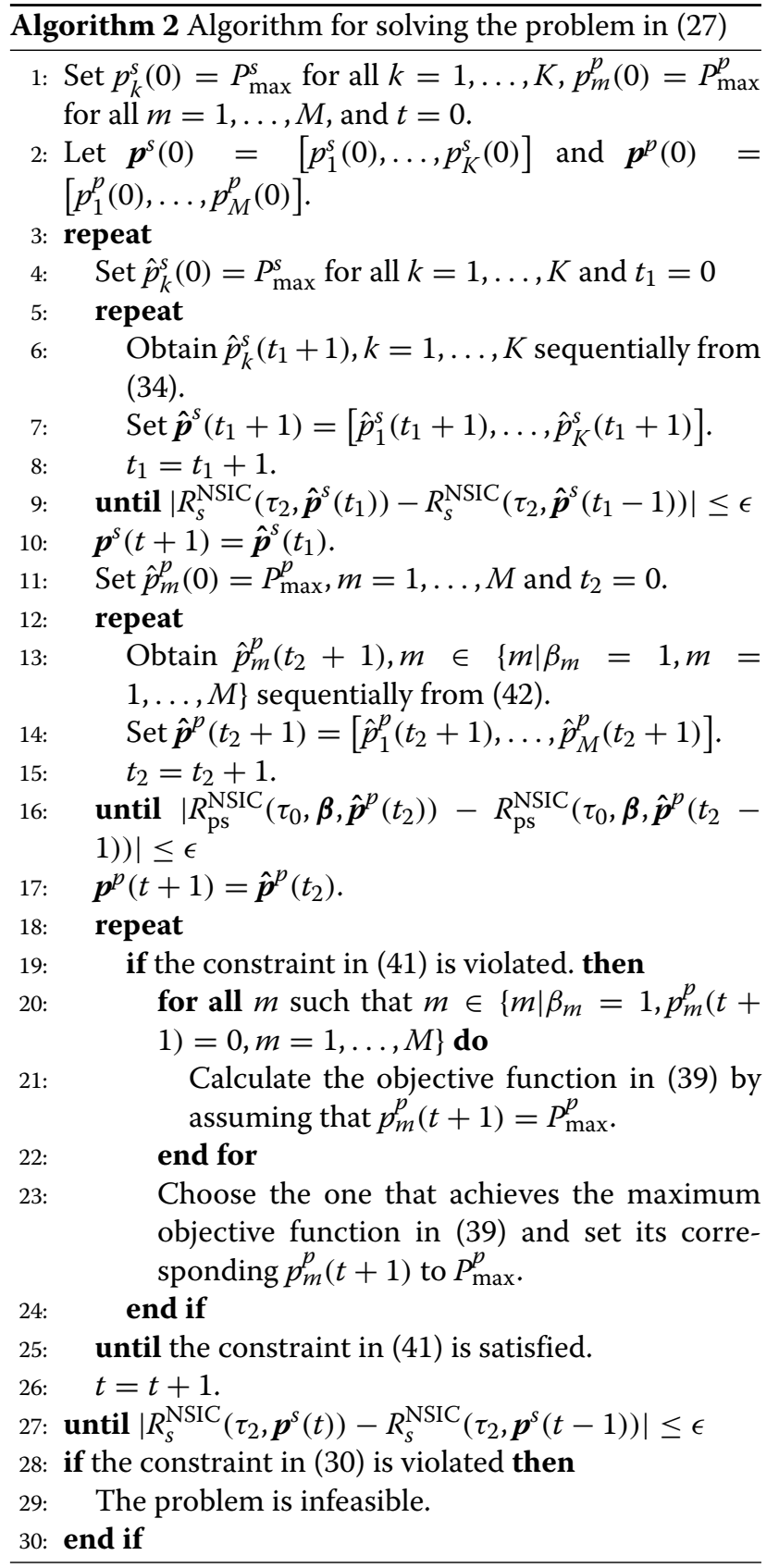

where $\quad C_{p}^{\mathrm{SIC}} \quad=\quad \ln \left(1+\frac{\sum_{m=1}^{M} \alpha_{m} p_{m}^{p} h_{m}^{p}}{\sigma^{2}}\right)+$ $\ln \left(1+\frac{\sum_{m=1}^{M} \beta_{m} p_{m}^{p} h_{m}^{\mathrm{ps}}}{\sigma^{2}}\right)$. The above problem belongs to the linear programming and thus can be solved efficiently by the simplex method [34].

With given $\tau_{0}, \tau_{1}, \tau_{2}, \boldsymbol{p}^{p}, \boldsymbol{p}^{s}$, we optimize $\boldsymbol{\alpha}, \boldsymbol{\beta}$ as given by

$$
\begin{aligned}
& \max _{\boldsymbol{\alpha}, \boldsymbol{\beta}} R_{s}^{\mathrm{SIC}}\left(\tau_{2}, \boldsymbol{p}^{s}\right) \\
& \text { s.t. } \alpha_{m} \in\{0,1\}, \beta_{m} \in\{0,1\}, \forall m,
\end{aligned}
$$

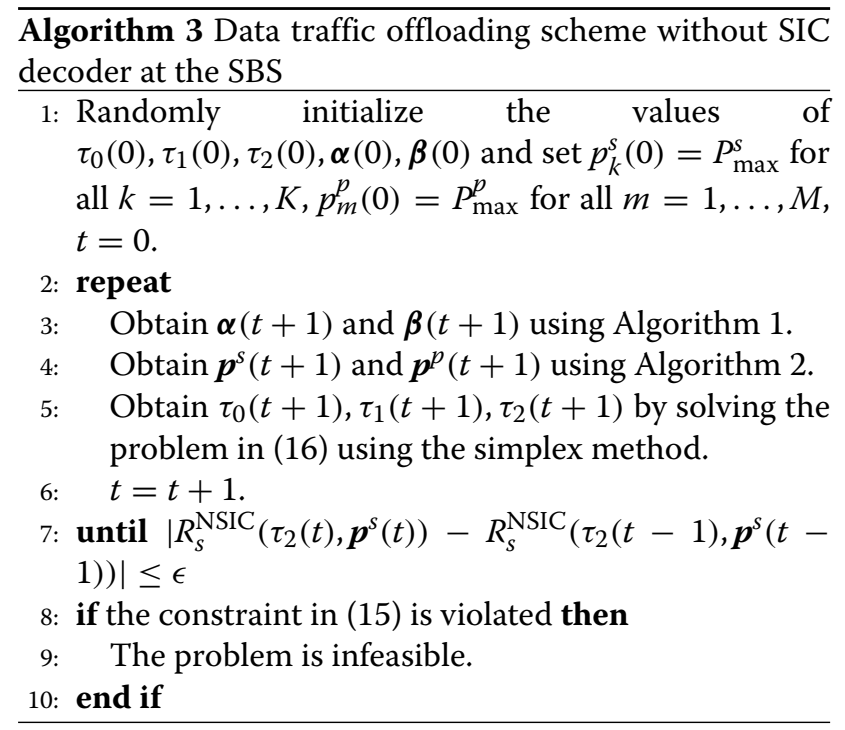

The above problem can be solved similar to the problem in (20) and the algorithm is listed in Algorithm 4.

With given $\tau_{0}, \tau_{1}, \tau_{2}, \boldsymbol{\alpha}, \boldsymbol{\beta}$, we optimize $\boldsymbol{p}^{p}, \boldsymbol{p}^{s}$ as given by

$$
\begin{aligned}
& \max _{\boldsymbol{p}^{p}, \boldsymbol{p}^{s}} R_{s}^{\mathrm{SIC}}\left(\tau_{2}, \boldsymbol{p}^{s}\right) \\
& \text { s.t. } 0 \leq p_{m}^{p} \leq P_{\max }^{p}, \forall m,
\end{aligned}
$$

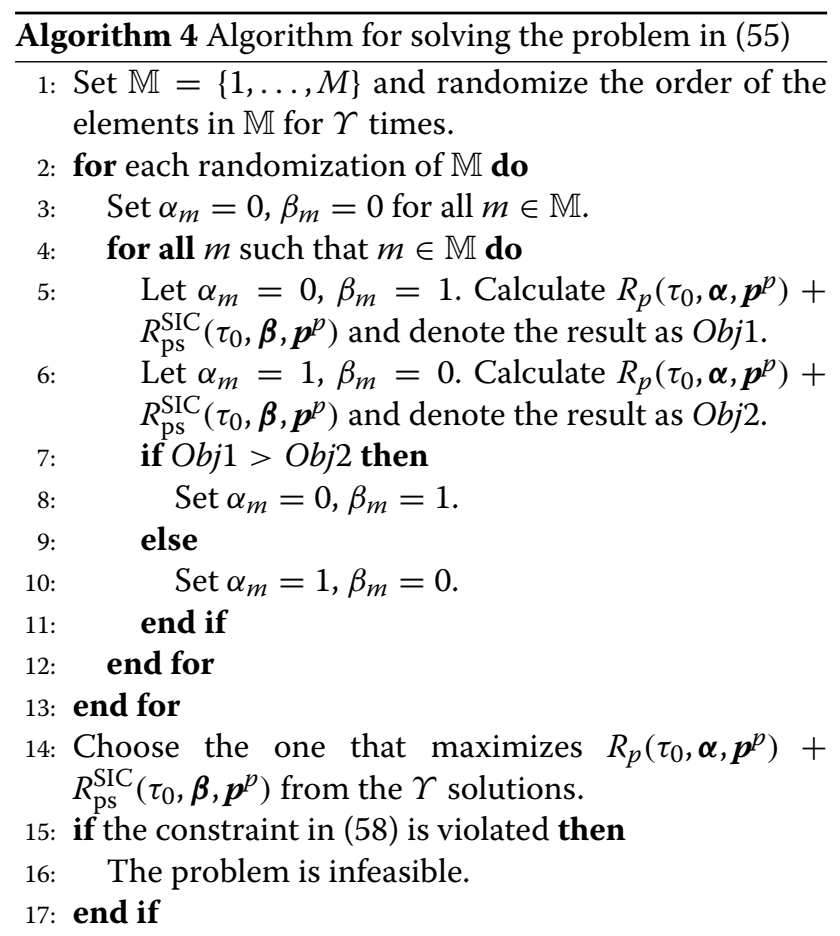




$$
\begin{aligned}
& 0 \leq p_{k}^{s} \leq \frac{\zeta \tau_{0} \sum_{m=1}^{M} p_{m}^{p} h_{m, k}+\zeta \tau_{1} p_{\mathrm{SBS}}^{s} h_{k}^{s}}{\tau_{2}}, \forall k \\
& R_{p}\left(\tau_{0}, \boldsymbol{\alpha}, \boldsymbol{p}^{p}\right)+R_{\mathrm{ps}}^{\mathrm{SIC}}\left(\tau_{0}, \boldsymbol{\beta}, \boldsymbol{p}^{p}\right) \geq R_{\min } .
\end{aligned}
$$

The above problem can be solved by iteratively optimizing $\boldsymbol{p}^{s}$ with given $\boldsymbol{p}^{p}$ and optimizing $\boldsymbol{p}^{p}$ with given $\boldsymbol{p}^{s}$. With given $\boldsymbol{p}^{p}$, the value of $\boldsymbol{p}^{s}$ is optimized as

$$
\begin{aligned}
& \max _{\boldsymbol{p}^{s}} R_{s}^{\mathrm{SIC}}\left(\tau_{2}, \boldsymbol{p}^{s}\right) \\
& \text { s.t. } 0 \leq p_{k}^{s} \leq \frac{\zeta \tau_{0} \sum_{m=1}^{M} p_{m}^{p} h_{m, k}+\zeta \tau_{1} p_{\mathrm{SBS}}^{s} h_{k}^{s}}{\tau_{2}}, \forall k .
\end{aligned}
$$

From (8), it is seen that $R_{s}^{\mathrm{SIC}}\left(\tau_{2}, \boldsymbol{p}^{s}\right)$ is an increasing function of $p_{k}^{s}$. Thus, the solution to the problem in (63) is $p_{k}^{s}=\frac{\zeta \tau_{0} \sum_{m=1}^{M} p_{m}^{p} h_{m, k}+\zeta \tau_{1} p_{\mathrm{SBS}}^{s} h_{k}^{s}}{\tau_{2}}$ for $k=1, \ldots, K$. With given $\boldsymbol{p}^{s}$, we optimize $\boldsymbol{p}^{p}$ as given by

$$
\begin{aligned}
\max _{\boldsymbol{p}^{p}} & R_{s}^{\mathrm{SIC}}\left(\tau_{2}, \boldsymbol{p}^{s}\right) \\
\text { s.t. } & 0 \leq p_{m}^{p} \leq P_{\mathrm{max}}^{p}, \forall m, \\
& \sum_{m=1}^{M} p_{m}^{p} h_{m, k} \geq \frac{p_{k}^{s} \tau_{2}-\zeta \tau_{1} p_{\mathrm{SBS}}^{s} h_{k}^{s}}{\zeta \tau_{0}}, \forall k, \\
& R_{p}\left(\tau_{0}, \boldsymbol{\alpha}, \boldsymbol{p}^{p}\right)+R_{\mathrm{ps}}^{\mathrm{SIC}}\left(\tau_{0}, \boldsymbol{\beta}, \boldsymbol{p}^{p}\right) \geq R_{\min } .
\end{aligned}
$$

Since the objective function of the above problem does not depend on $\boldsymbol{p}^{p}$, the above problem can be solved by checking whether the problem is feasible by solving the following problem as

$$
\begin{aligned}
\max _{\boldsymbol{p}^{p}} & R_{p}\left(\tau_{0}, \boldsymbol{\alpha}, \boldsymbol{p}^{p}\right)+R_{\mathrm{ps}}^{\mathrm{SIC}}\left(\tau_{0}, \boldsymbol{\beta}, \boldsymbol{p}^{p}\right) \\
\text { s.t. } 0 \leq p_{m}^{p} \leq P_{\max }^{p}, \forall m, & \forall{ }^{M} p_{m=1}^{p} h_{m, k} \geq \frac{p_{k}^{s} \tau_{2}-\zeta \tau_{1} p_{\mathrm{SBS}}^{s} h_{k}^{s}}{\zeta \tau_{0}}, \forall k .
\end{aligned}
$$

The above problem is convex but does not have a closedform solution. Therefore, the interior point method [35] can be used to solve the above problem and we omit the details here for brevity. The algorithm to solve the problem in (59) is listed in Algorithm 5.

The overall data traffic offloading scheme with SIC decoder at the SBS is listed in Algorithm 6.

\section{Simulation results}

This section verifies the performance of the proposed data traffic offloading schemes using simulations. In the following results, we assume that all the channels involved follow Rayleigh fading with unit mean and set $\sigma^{2}=1$, $T=1, M=10$, and $K=10$.

Figure 2 plots the MUE sum rate against the required minimum MUE sum rate $R_{\min }$ for different values of $P_{\max }^{p}$. The results from the scheme without data traffic offloading are also given for the purpose of comparison. It is
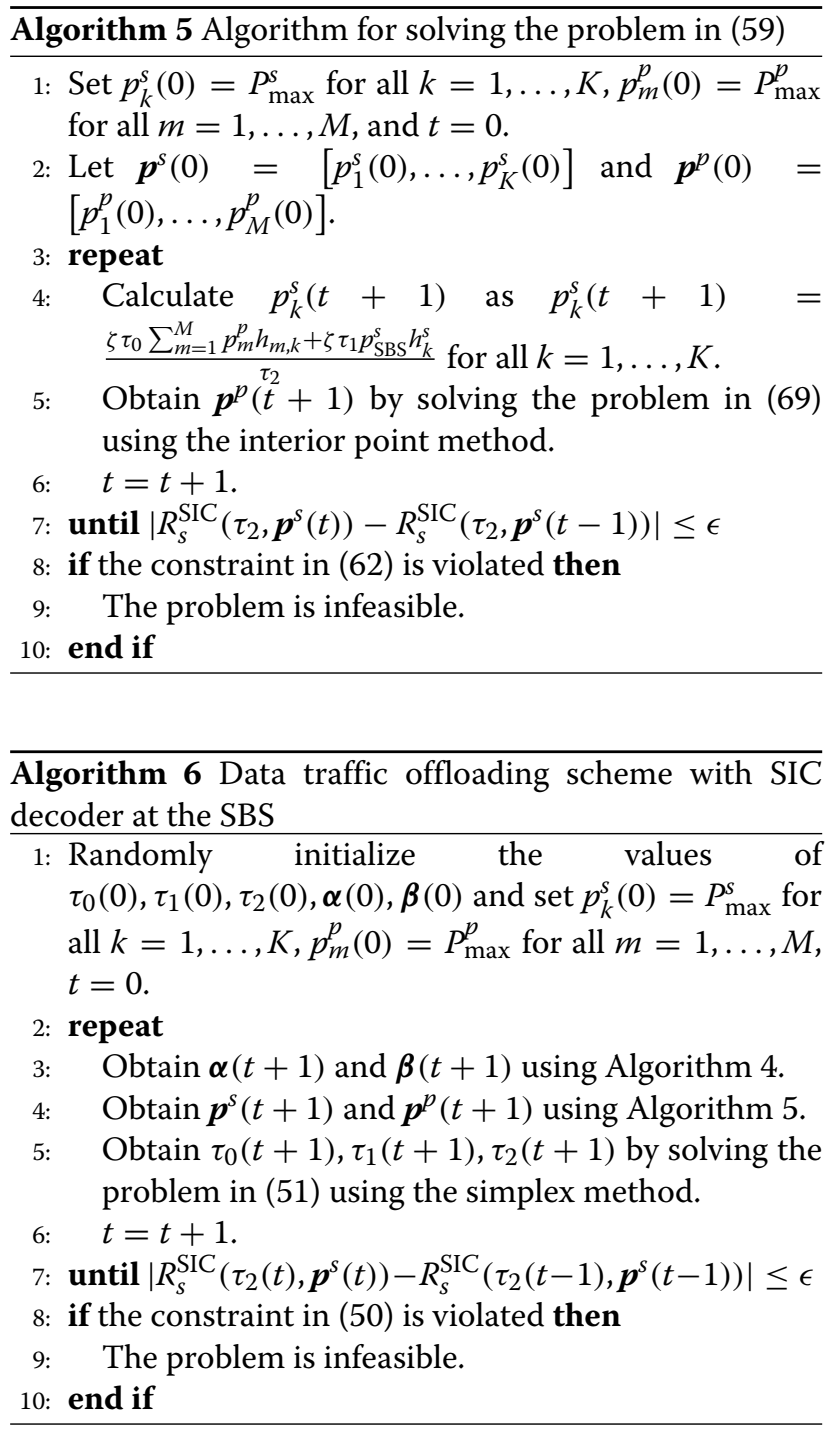

seen that the proposed data offloading schemes achieve much higher MUE sum rate than the scheme without data offloading. It is also seen that the proposed data offloading scheme with SIC at the SBS achieves much higher MUE sum rate than the scheme without SIC at the SBS, especially when $R_{\min }$ is large. In addition, it is seen that the MUE sum rate increases as $R_{\min }$ increases until saturates to a certain level. Especially, it is seen that for a small $R_{\min }$, the achieved MUE sum rate is equal to $R_{\min }$, while for a large $R_{\min }$, the achieved MUE sum rate is lower than $R_{\min }$. This is due to the fact that, if $R_{\min }$ is too high, the required minimum MUE sum rate cannot be achieved even if all the available resources are allocated to the MUEs with data offloading provided by the SBS. Furthermore, it is seen that the MUE sum rate increases as $P_{\max }^{p}$ increases, especially when $R_{\min }$ is large.

Figure 3 plots the SUE sum rate against the required minimum MUE sum rate $R_{\min }$ for different values of 


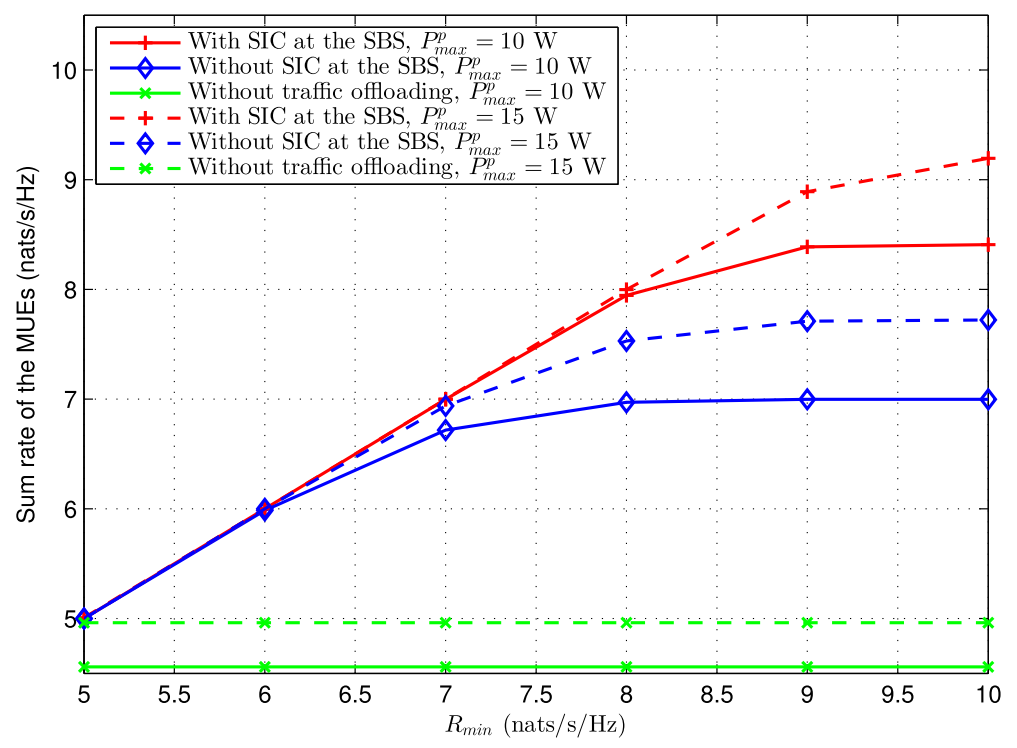

Fig. 2 MUE sum rate against $R_{\text {min }}$

$P_{\max }^{p}$. It is seen that the SUE sum rate decreases as $R_{\min }$ increases. This is due to the fact that less resources are remaining for the SUEs as $R_{\min }$ increases. It is also seen that the proposed data offloading scheme with SIC at the SBS outperforms the scheme without SIC at the SBS in terms of the SUE sum rate. In addition, it is seen that increasing $P_{\max }^{p}$ leads to higher SUE sum rate. This indicates that increasing $P_{\max }^{p}$ is beneficial to the SUEs.

Figure 4 plots the MUE sum rate against the maximum transmit power limit of the MUE $P_{\max }^{p}$ for different values of $R_{\min }$. It is seen that when $R_{\min }$ is small, the MUE sum rate keeps constant and is equal to $R_{\min }$ as $P_{\max }^{p}$ increases. This indicates that the proposed schemes can well guarantee the QoS of the MUEs. It is also seen that when $R_{\min }$ is large, the MUE sum rate achieved by the proposed scheme without SIC is lower than $R_{\min }$ and increases as $P_{\max }^{p}$ increases until saturates to the $R_{\mathrm{min}}$, while the MUE sum rate achieved by the proposed scheme with SIC keeps constant and is equal to $R_{\min }$ as $P_{\max }^{p}$ increases. This indicates that although the proposed scheme can not guarantee the QoS of the MUEs when $R_{\min }$ is very large, increasing $P_{\max }^{p}$

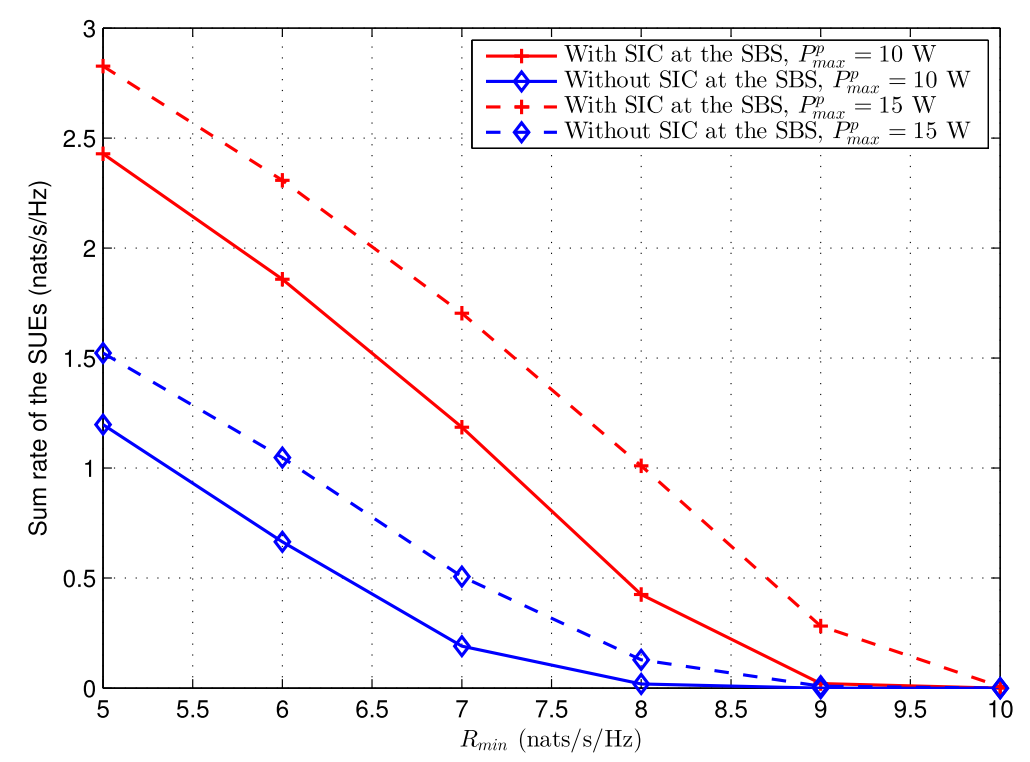

Fig. 3 SUE sum rate against $R_{\min }$ 


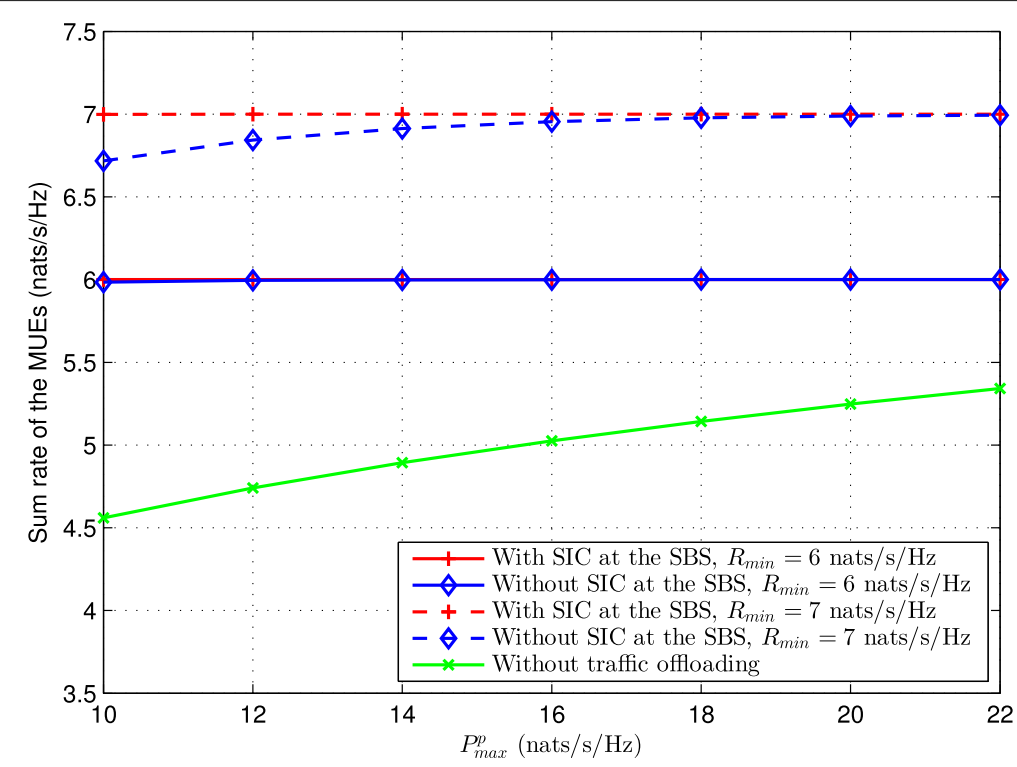

Fig. 4 MUE sum rate against $P_{\max }^{p}$

can let the proposed scheme achieve the MUE sum rate equal to $R_{\min }$.

Figure 5 plots the SUE sum rate against the maximum transmit power limit of the MUE $P_{\max }^{p}$ for different values of $R_{\min }$. It is seen that the SUE sum rate increases as $P_{\max }^{p}$ increases. This indicates that higher value of $P_{\max }^{p}$ is beneficial to the SUEs. It is also seen that the SUE sum rate with SIC is higher than that without SIC and the gap between them is almost constant as $P_{\max }^{p}$ increases. In addition, it is seen that the SUE sum rate with lower $R_{\min }$ is higher than that with higher $R_{\min }$ and the gap between them is nearly unchanged as $P_{\max }^{p}$ increases.

\section{Conclusions}

We consider a third-party cognitive small cell with wireless powered SUEs that processes data traffic offloaded from a primary macrocell. As long as the QoS of the primary macrocell is satisfied, the cognitive small cell is assumed to be able to use remaining resources for its own purpose. Iterative optimization-based data traffic

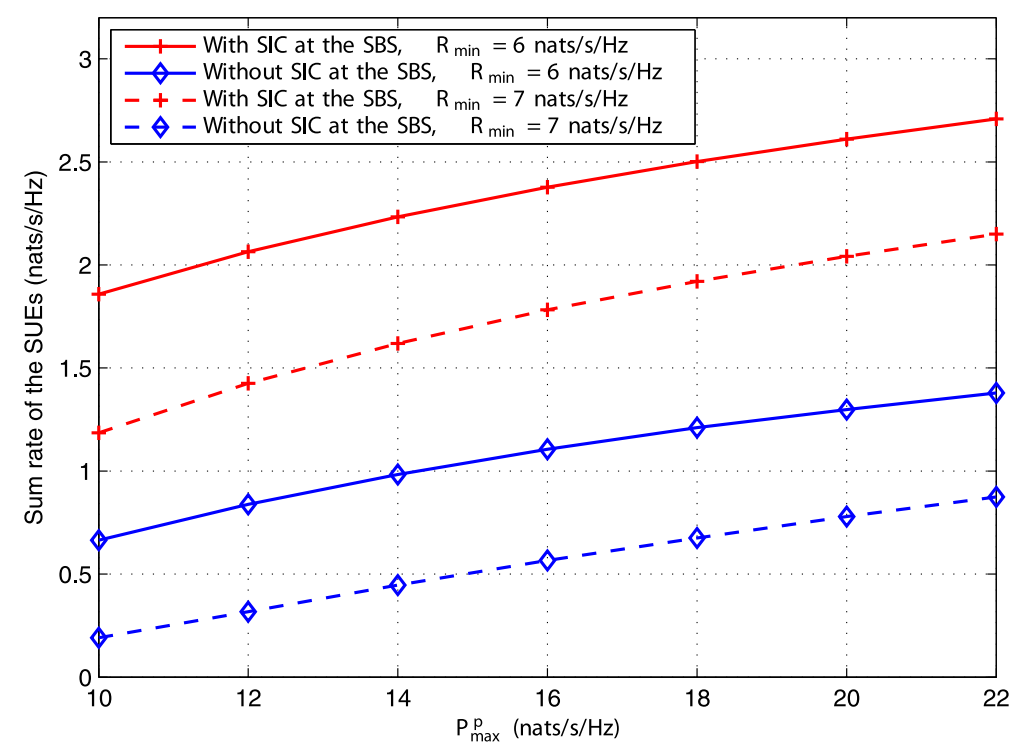

Fig. 5 SUE sum rate against $P_{\max }^{D}$ 
offloading schemes with SIC decoder available or not available at the SBS are proposed to maximize the SUE sum rate under the required minimum MUE sum rate constraint. We show that the proposed data traffic offloading schemes are effective in improving the performance of the MUEs and providing transmission opportunities for the wireless powered SUEs.

\section{Endnotes}

${ }^{1}$ The case of multi-band is not considered in this paper, and we leave it in our future work.

${ }^{2}$ We assume that there are multiple heterogeneous services carried by each MUE. Some services are real-time and the other services are non-real-time, while some services generate continuous data and the other services generate burst data. Thus, simply restricting the minimum rate for each individual MUE is inappropriate for guaranteeing QoS of the MUEs. So, we try to guarantee the minimum sum rate for all the MUEs to provide satisfactory QoS to the MUEs. By satisfying such required minimum MUE sum rate, the QoS of the MUEs can be satisfied on a long-term basis. The value of $R_{\min }$ can be chosen based on long-term measurements of the data rate requirements of the MUEs.

\section{Acknowledgements}

This work was supported in part by the National Natural Science Foundation of China under grant no. 61401218 and in part by the National Science and Technology Major Project of China under grant no. 2017ZX03001008.

\section{Authors' contributions}

DX proposed the idea of this paper and wrote the paper. QL performed the simulations. Both authors read and approved the final manuscript.

\section{Competing interests}

The authors declare that they have no competing interests.

\section{Publisher's Note}

Springer Nature remains neutral with regard to jurisdictional claims in published maps and institutional affiliations.

Received: 25 July 2017 Accepted: 6 November 2017

Published online: 21 November 2017

\section{References}

1. F Rebecchi, MD de Amorim, V Conan, A Passarella, R Bruno, M Conti, Data offloading techniques in cellular networks: a survey. IEEE Commun. Surv. Tutor. 17(2), 580-603 (2015)

2. S Haykin, Cognitive radio: brain-empowered wireless communications. IEEE J. Sel. Areas Commun. 23(2), 201-220 (2005)

3. H Zhang, C Jiang, NC Beaulieu, X Chu, X Wen, M Tao, Resource allocation in spectrum-sharing OFDMA femtocells with heterogeneous services. IEEE Trans. Commun. 62(7), 2366-2377 (2014)

4. H Zhang, C Jiang, NC Beaulieu, X Chu, X Wang, TQ Quek, Resource allocation for cognitive small cell networks: a cooperative bargaining game theoretic approach. IEEE Trans. Wirel. Commun. 14(6), 3481-3493 (2015)

5. H Zhang, C Jiang, X Mao, H-H Chen, Interference-limited resource optimization in cognitive femtocells with fairness and imperfect spectrum sensing. IEEE Trans. Veh. Technol. 65(3), 1761-1771 (2016)
6. D Xu, Q Li, Resource allocation for outage probability minimisation in cognitive radio multicast networks. Trans. Emerg. Telecommun. Technol. 27(1), 51-63 (2016)

7. D Xu, Q Li, Power allocation for two-user cognitive multiple access channels under primary user outage constraint. Int. J. Commun. Syst. 30(5) (2017). doi:10.1002/dac.3096

8. H Zhang, Y Nie, J Cheng, VC Leung, A Nallanathan, Sensing time optimization and power control for energy efficient cognitive small cell with imperfect hybrid spectrum sensing. IEEE Trans. Wirel. Commun. 16(2), 730-743 (2017)

9. $\mathrm{D} X \mathrm{u}, \mathrm{Q} \mathrm{Li}$, Resource allocation for cognitive radio with primary user secrecy outage constraint. IEEE Syst. J (2017). doi:10.1109/JSYST.2016.2585654

10. D Xu, Q Li, Improving physical-layer security for primary users in cognitive radio networks. IET Commun (2017). doi:10.1049/iet-com.2017.0323

11. M-L Ku, W Li, Y Chen, KR Liu, Advances in energy harvesting communications: past, present, and future challenges. IEEE Commun. Surv. Tutor. 18(2), 1384-1412 (2016)

12. Y Sun, XXu, R Zhang, R Gao, in Proc. International Symposium on Wireless Personal Multimedia Communications. Offloading based load balancing for the small cell heterogeneous network (IEEE, Sydney, 2014), pp. 288-293

13. P Trakas, F Adelantado, C Verikoukis, in Proc. IEEE International Conference on Communications. A novel learning mechanism for traffic offloading with small cell as a service (IEEE, London, 2015), pp. 6893-6898

14. XXu, H Zhang, X Dai, X Tao, in Proc. International Conference on Communications and Networking in China. Optimal Energy Efficient Offloading in small cell HetNet with auction (Springer, Shanghai, 2014), pp. $335-340$

15. BH Jung, NO Song, DK Sung, A network-assisted user-centric WiFi-offloading model for maximizing per-user throughput in a heterogeneous network. IEEE Trans. Veh. Technol. 63(4), 1940-1945 (2014)

16. D Suh, H Ko, S Pack, Efficiency analysis of WiFi offloading techniques. IEEE Trans. Veh. Technol. 65(5), 3813-3817 (2016)

17. P Luong, TM Nguyen, LB Le, ND Dao, E Hossain, Energy-efficient WiFi offloading and network management in heterogeneous wireless networks. IEEE Access. 4, 10210-10227 (2016)

18. P GroNsund, O GroNdalen, M LaHteenoja, Business case evaluations for LTE network offloading with cognitive femtocells. Telecommun. Policy. 37(2), 140-153 (2013)

19. G Mange, U Celentano, PH Lehne, T Tjeltas, M Lopez-Benitez, in Proc. Future Network and Mobile Summit. Cognitive architecture and system solutions to offload LTE networks in TVWS (IEEE, Lisbon, 2013), pp. 1-8

20. D Liu, Y Chen, KK Chai, L Cuthbert, T Zhang, in Proc. International Conference on Communications and Networking in China. Cognitive cooperative traffic offloading scheme over heterogeneous networks (IEEE, Kunming, 2012), pp. 737-741

21. H ElSawy, E Hossain, DI Kim, HetNets with cognitive small cells: user offloading and distributed channel access techniques. IEEE Commun. Mag. 51(6), 28-36 (2013)

22. KXiong, P Fan, C Zhang, KB Letaief, Wireless information and energy transfer for two-hop non-regenerative MIMO-OFDM relay networks. IEEE J. Sel. Areas Commun. 33(8), 1595-1611 (2015)

23. Y Dong, $\mathrm{H}$ Zhang, MJ Hossain, J Cheng, VC Leung, in Proc. IEEE Global Communications Conference. Energy efficient resource allocation for OFDM full duplex distributed antenna systems with energy recycling (IEEE, San Diego, 2015), pp. 1-6

24. H Zhang, J Du, J Cheng, VC Leung, in Proc. IEEE Global Communications Conference. Resource allocation in SWIPT enabled heterogeneous cloud small cell networks with incomplete CSI (IEEE, Washington, DC, 2016), pp. 1-5

25. KXiong, C Chen, G Qu, P Fan, KB Letaief, Group cooperation with optimal resource allocation in wireless powered communication networks. IEEE Trans. Wirel. Commun. 16(6), 3840-3853 (2017)

26. H Zhang, S Huang, C Jiang, K Long, VC Leung, HV Poor, Energy efficient user association and power allocation in millimeter-wave-based ultra dense networks with energy harvesting base stations. IEEE J. Sel. Areas Commun. 35(9), 1936-1947 (2017)

27. Y Dong, Z Chen, P Fan, Capacity region of gaussian multiple-access channels with energy harvesting and energy cooperation. IEEE Access. 5 1570-1578 (2017)

28. D Xu, Q Li, Price-based time and energy allocation in cognitive radio multiple access networks with energy harvesting. Sci. China Information Sci. 60(10), 108302 (2017) 
29. M Pratibha, KH Li, KC Teh, Channel selection in multichannel cognitive radio systems employing RF energy harvesting. IEEE Trans. Veh. Technol. 65(1), 457-462 (2016)

30. D Xu, Q Li, Joint power control and time allocation for wireless powered underlay cognitive radio networks. IEEE Wirel. Commun. Lett. 6(3), 294-297 (2017)

31. S Yin, Z Qu, Z Wang, L Li, Energy-efficient cooperation in cognitive wireless powered networks. IEEE Commun. Lett. 21(1), 128-131 (2017)

32. $\mathrm{D} X \mathrm{u}, \mathrm{Q} \mathrm{Li}, \mathrm{Cooperative} \mathrm{resource} \mathrm{allocation} \mathrm{in} \mathrm{cognitive} \mathrm{radio} \mathrm{networks}$ with wireless powered primary users. IEEE Wirel. Commun. Lett (2017). doi:10.1109/LWC.2017.2727493

33. S Lee, R Zhang, Cognitive wireless powered network: spectrum sharing models and throughput maximization. IEEE Trans. Cogn. Commun. Netw. 1(3), 335-346 (2015)

34. DG Luenberger, Introduction to linear and nonlinear programming, vol. 28. (Addison-Wesley Reading, MA, USA, 1973)

35. S Boyd, L Vandenberghe, Convex optimization. (Cambridge Univ. Press, Cambridge, 2004)

\section{Submit your manuscript to a SpringerOpen ${ }^{\circ}$ journal and benefit from:}

- Convenient online submission

Rigorous peer review

- Open access: articles freely available online

- High visibility within the field

- Retaining the copyright to your article

Submit your next manuscript at $>$ springeropen.com 